\title{
DỨBin
}

Technological University Dublin ARROW@TU Dublin

\section{Directional Modulation for Compact Devices}

\author{
Adam Narbudowicz \\ Technological University Dublin, adam.narbudowicz@mydit.ie
}

Follow this and additional works at: https://arrow.tudublin.ie/ahfrcart

Part of the Systems and Communications Commons

\section{Recommended Citation}

Narbudowicz, A., Ammann, M.J. \& Heberling, D. (2017). Directional Modulation for Compact Devices. IEEE Antennas and Wireless Propagation Letters, vol. PP, no. 99, 2017. doi:10.1109/LAWP.2017.2697720

This Article is brought to you for free and open access by the Antenna \& High Frequency Research Centre at ARROW@TU Dublin. It has been accepted for inclusion in Articles by an authorized administrator of ARROW@TU Dublin. For more information, please contact arrow.admin@tudublin.ie, aisling.coyne@tudublin.ie, gerard.connolly@tudublin.ie.

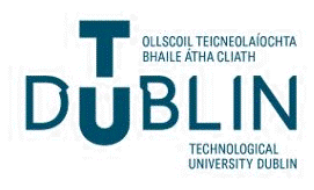




\title{
Directional Modulation for Compact Devices
}

\author{
Adam Narbudowicz, Member, IEEE, Max J. Ammann, Senior Member, IEEE, and Dirk Heberling, \\ Senior Member, IEEE
}

\begin{abstract}
A new directional modulation system which uses radiation pattern data for modulation encoding is proposed. The approach allows compact multiport antennas (e.g. MIMO capable antennas) to be used for directional modulation, replacing extensive arrays. The modulation weighting coefficients are calculated with a fixed, antenna specific equation, thus reducing computational complexity. The solution is intended to enhance privacy in small, battery-operated wireless devices, required for "Internet of Things" applications.

Index Terms-smart antenna, directional modulation, physical layer security, Internet of Things
\end{abstract}

\section{INTRODUCTION}

$\mathrm{T}$ HE forthcoming implementation of "Smart Cities" and "Internet of Things" (IoT) will see proliferation of wireless connections to sensors and daily-use devices. Consequently, highly sensitive personal data will be present in the open wireless medium. Even partial interception of such data may lead to devastating consequences for individuals or even societies, opening-up multiple abuse possibilities. Thus the need for secure communication is greater than before. However many IoT implementations face physical constraints due to the need for small, cost efficient modules, limited by battery performance and low computational power. Due to necessity, some manufacturers attempt to use unsecure bespoke or customized protocols, while others strip standard protocols from certain safety features [1] - both methods resulting in vulnerable communications.

To provide strong security, good cryptography protocols are required. However the proposed use of radio hardware can provide an additional layer of privacy, capable of impeding data interception. One technique investigated for this purpose is Directional Modulation (DM) [2-6], which in its most common implementation requires a large and bulky antenna array with a complex weighting system. Some simplification was proposed by Tao Hong et al. in [6], where a two-antenna system is used with antenna ports connected directly to the I and Q outputs of the standard QAM transceiver. Although the solution is costand power-efficient, it still requires an antenna array with a certain spacing to transmit the signal.

In [7] a compact antenna was proposed for $360^{\circ}$ beamsteering. To allow this functionality, the antenna

Manuscript received on December $29^{\text {th }}, 2016$. This work was supported by Irish Research Council, grant no ELEVATEPD/2014/79.

A. Narbudowicz and M. J. Ammann are with the Dublin Institute of Technology, Dublin 8, Ireland (e-mail: adam.narbudowicz@dit.ie).

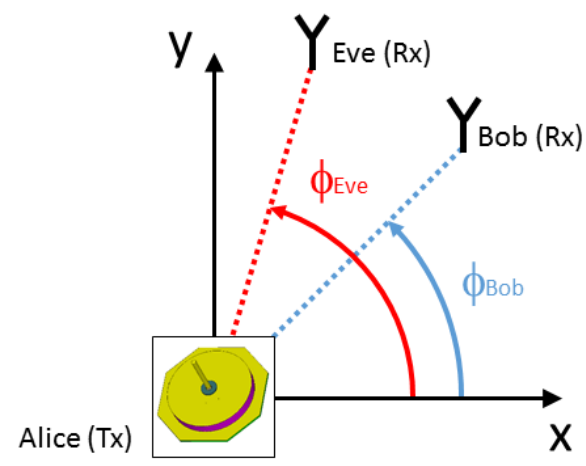

Fig. 1. Coordinate system used throughout the paper. Alice (transmitter) uses antenna proposed in [7], while Bob and Eve may use any antenna.

introduces a spatial variation of its radiation pattern phase, i.e. a signal sent in the $\phi$ direction has a phase shift roughly proportional to $2 \phi$. This not only allows a beam steering capability, but combined with a linear array offers more flexibility to shape the desired pattern [8].

This paper demonstrates the first implementation of DM with a compact antenna. Unlike other approaches, it relies on a newly proposed antenna with a phase variation across its main beam (in this example an omnidirectional pattern) and uses the complex radiation pattern data for the DM coding. Distinct from previous works, the weights for directional modulation are calculated based on complex radiation patterns of the antenna. This allows a substantial size reduction and consequently operation from a spatially limited platform. Although the technique can be also implemented for larger arrays, it is intended as a compact and simple low-cost solution for enhanced privacy in future "Internet of Things" wireless communication that is power efficient, requiring little computational complexity and small antenna size. The proposed solution is the first that allows DM without the need for a large antenna array.

It should be noted that the proposed work demonstrates a different concept to [5], which uses only a simple phase shifter to control the direction of DM, without considering the antenna radiation patterns. Incorporation of the radiation patterns into the modulation technique provides a more generic solution, more robust to manufacturing inaccuracies, offers more precise control of the direction $\phi_{B o b}$ and allows the use of amplitude variations, in addition to phase, to scramble the modulation

D. Heberling is with the Institute of High Frequency Technology, RWTH Aachen University, 52074 Aachen, Germany. 
plane.

Throughout the paper we will use terminology and the coordinate system as shown in Fig. 1. In the center of the coordinate system there is a transmitter which uses the proposed DM scheme with a switchless reconfigurable antenna [7]. This transmitter will be denoted as Alice. The intended recipient of the message will be denoted as Bob and is located at an angle $\phi_{B o b}$ with respect to Alice. The eavesdropper will be denoted as Eve and is located at $\phi_{E v e}$ with respect to Alice. There are no limitations imposed on the antennas used by either Bob or Eve.

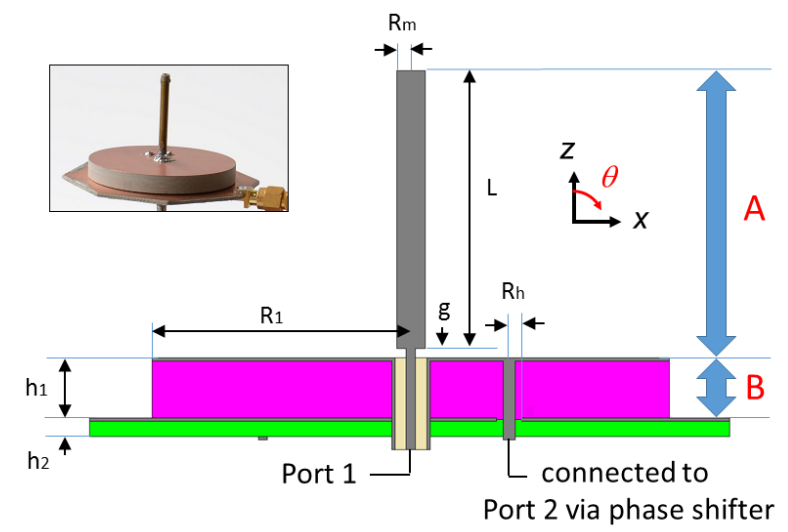

Fig. 2. Antenna used for the proposed directional modulation.

\section{ANTENNA}

The antenna used for the proposed technique is depicted in Fig 2. It comprises of two radiators, denoted as A and $\mathrm{B}$. The top-most (A) is a standard quarter-wavelength monopole, connected via a semi-rigid coaxial line to port 1 . Below (B) is located a dielectric-loaded patch, operating with two orthogonal $\mathrm{TM}_{21}$ modes excited with $90^{\circ}$ phase shift. This generates a rotating electric field, producing phase variation of the radiation pattern (seen in Fig. 3b). The antenna is discussed in more detail in [7].

The antenna was manufactured and measured. The $S_{11}$ in the $2.5 \mathrm{GHz}$ band is $-12 \mathrm{~dB}$ for port 1 and $-17 \mathrm{~dB}$ for port 3 , with isolation better than $18 \mathrm{~dB}$ [7]. The phase and amplitude radiation patterns in the $x z$-plane (dominant horizontal polarization) are shown in Fig 3. It can be seen, that while the monopole A generates a signal with constant phase-shift around $0^{\circ}$, part B produces a signal with a phase linearly dependent on the angle $\phi$. This property is a key feature which allows large arrays to be replaced by a small antenna structure in DM applications. In general, the proposed technique is expected to be effective with any two antenna types, which exhibit sufficient phase variation between their radiation patterns.

The antenna dimensions are: $L=28 \mathrm{~mm} ; R_{m}=1.5 \mathrm{~mm}$; $R_{l}=26 \mathrm{~mm} ; g=2 \mathrm{~mm} ; h_{l}=6 \mathrm{~mm} ; h_{2}=1.5 \mathrm{~mm} ; R_{h}=2 \mathrm{~mm}$.

\section{DIRECTIONAL MODULATION}

Unlike previous work, the proposed technique combines the antenna properties with a modulation scheme to execute simple DM without the need for computationally complex optimization. If the intended recipient (Bob) is located in the direction $\phi_{B o b}$, the DM might be realized by exciting the antenna

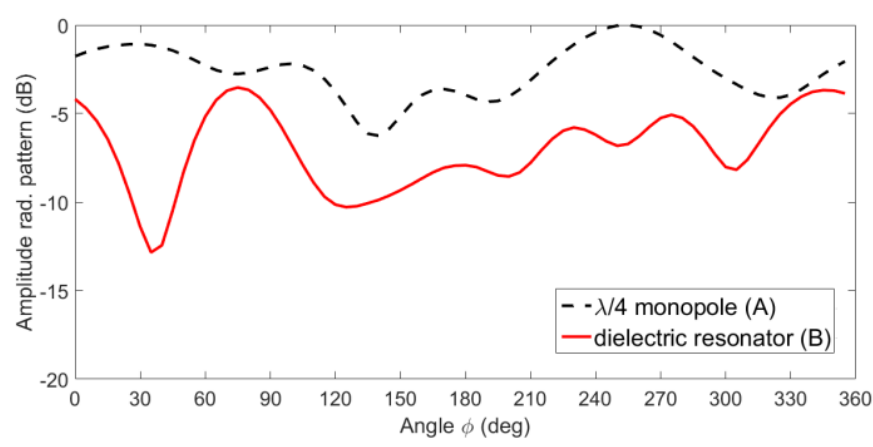

a)

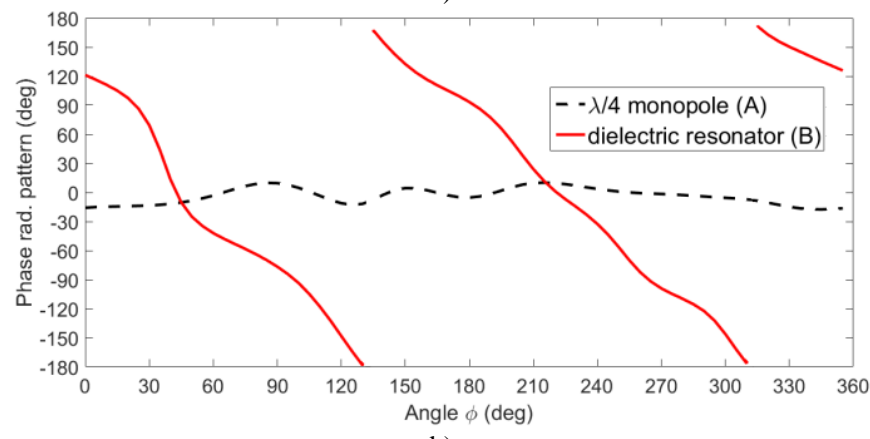

b)

Fig. 3. Measured radiation patterns of the proposed antenna in $x z$-plane $\left(\theta=90^{\circ}\right)$ at $2.5 \mathrm{GHz}$, vertical polarization: a) amplitude; b) phase.

ports 1 and 2 respectively with signals $T x_{1}$ and $T x_{2}$ defined as follows:

$T x_{1}=\frac{\operatorname{Re}(\vec{m})}{G_{A}\left(\phi_{B o b}\right)}$

$T x_{2}=\frac{j \operatorname{Im}(\vec{m})}{G_{B}\left(\phi_{B o b}\right)}$

where $\vec{m}$ is a complex modulation vector and $G_{A}(\phi), G_{B}(\phi)$ are complex (i.e. including phase delay) radiation patterns generated by the A and B elements respectively, as shown in Fig. 3. Please note, that in order to perform the proposed scheme the antenna has to be well characterized in an anechoic chamber - at least with respect to the plane, where the DM should occur.

The physical meaning of (1) is that the in-phase component of the original modulation vector is transmitted solely by the $\lambda / 4$ monopole (A), while the quadrature component of the original vector is transmitted solely by the dielectric-loaded patch (B). Due to the components $G_{A}\left(\phi_{B o b}\right)$ and $G_{B}\left(\phi_{B o b}\right)$ in (1), the modulation constellation will be seen unobstructed at $\phi_{B o b}$ while scrambled elsewhere. This also necessitates that every change in $\phi_{B o b}$ will yield change in the modulation scheme used.

Fig 4 illustrates the modulation vector trajectories on the complex modulation plane as a function of $\phi_{E v e}$ for 8-PSK. Four exemplary values of $\phi_{B o b}$ were used. It can be seen, that although the general performance is similar, there are indeed some variations with different $\phi_{B o b}$ values.

The 8-PSK modulation is chosen, because two points (i.e. on I axis) are transmitted solely by the $\lambda / 4$ monopole, two points (i.e. on $\mathrm{Q}$ axis) are transmitted solely be the dielectric-loaded patch and the four remaining are transmitted with a 

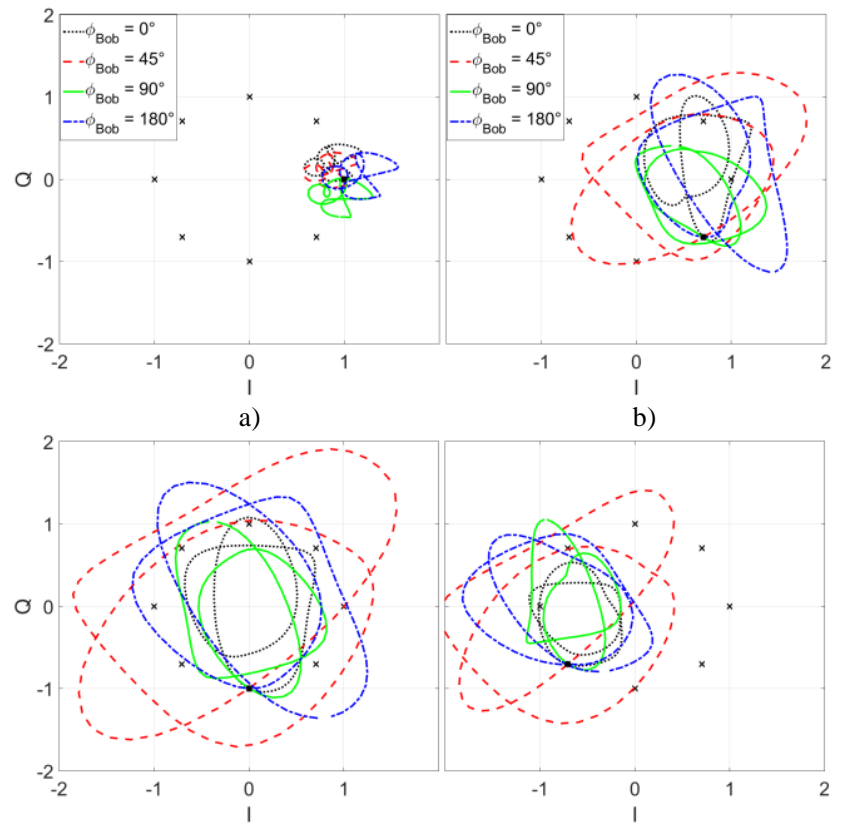

c)

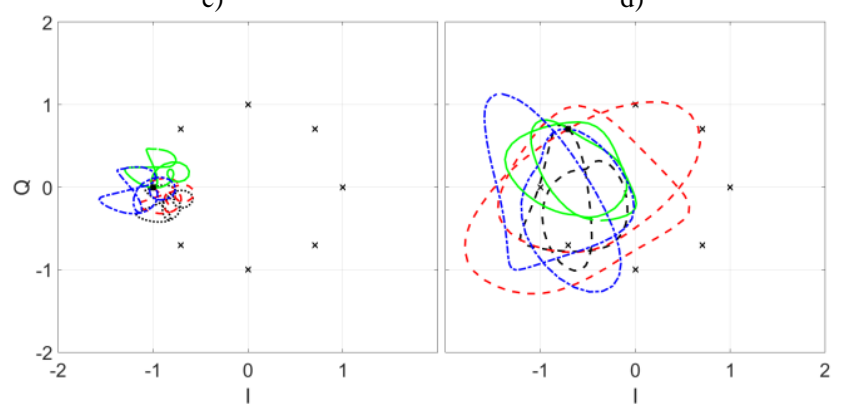

e)

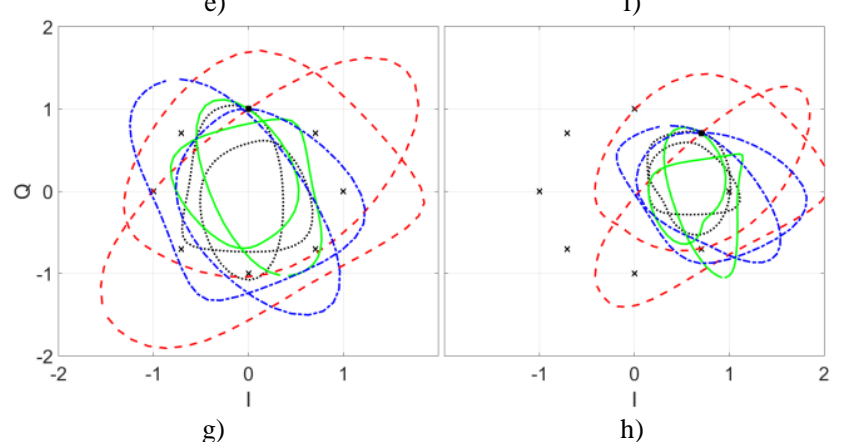

Fig. 4. Trajectories of modulation points in 8-PSK as a function of angle $\phi$ : a) phase $0^{\circ}$; b) phase $-45^{\circ}$; c) phase $-90^{\circ}$; d) phase $-135^{\circ}$; e) phase $180^{\circ}$; f) phase $135^{\circ}$; g) phase $90^{\circ}$; h) phase $45^{\circ}$.

superposition of both antennas. It can be seen, that as expected from Fig. 2, the points with phase $0^{\circ}$ and $180^{\circ}$ are almost constant, while $-90^{\circ}$ and $+90^{\circ}$ scribe a full circle due to the phase variation of the radiation pattern.

\section{RESULTS}

To demonstrate the proposed concept, a BER for four modulation configurations was calculated for various $\phi_{B o b}$ directions. The calculation was performed in Matlab and is based on real data, as measured in the spherical near-field anechoic chamber. The calculation assumes the strict condition, that Eve has full knowledge of the system, including the antenna orientation and radiation patterns. On the contrary, it is assumed that Bob has no knowledge of the DM system or antenna involved and expects to receive standard 4-PSK or 8PSK modulation.

\section{A. 4-PSK Modulation}

The proposed system was firstly tested with a 4-PSK modulation and a $12 \mathrm{~dB}$ Signal to Noise Ratio (SNR), where all four points are transmitted as a superposition of both radiators. This corresponds to the $\phi$-dependent trajectories seen in Figs. 3b), 3d), 3f) and 3h).

Since Bob has no information on the patterns $G_{A}$ and $G_{B}$ which are used to modulate the signal in (1), his BER is calculated with respect to the idealized 4-PSK constellation. On the contrary, for Eve we assume the worst possible scenario that she obtained the complex radiation patterns $G_{A}$ and $G_{B}$, thus

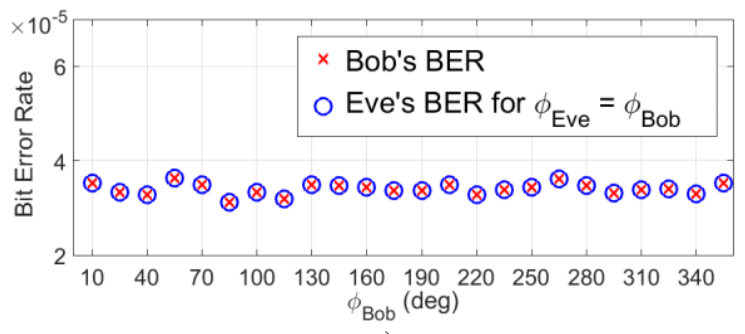

a)

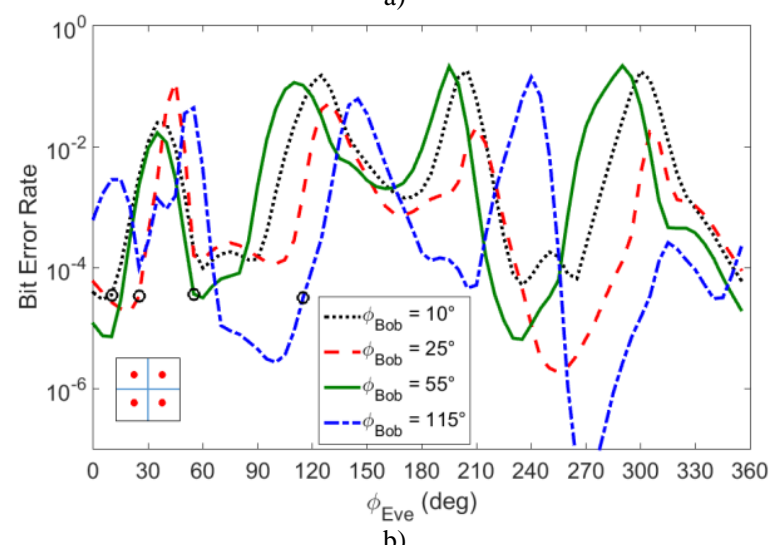

b)

Fig. 5. Bit error rate for the simple 4-PSK modulation: a) BER for Bob vs. Eve when $\phi_{\mathrm{Eve}}=\phi_{\mathrm{Bob}}$; b) Eve's BER as a function of direction $\phi_{\mathrm{Eve}}$ in four exemplary $\phi_{\text {Bob }}$ cases.

possesses full system knowledge and can partially compensate for the scrambling of the modulation points. Consequently, when $\phi_{B o b}=\phi_{E v e}$ the BER seen by Bob can be only as good or worse than that seen by Eve. This is a generally known limitation of all currently available DM implementations, although there are some mitigation research efforts [3].

Fig. 5a shows the BER for 24 different configurations, each generated for different directions $\phi_{B o b}$ in $15^{\circ}$ steps. The figure compares Bob and Eve's BER for the worst possible case when $\phi_{B o b}=\phi_{E v e}$ (i.e. Bob and Eve are located in the same direction). The BER for Eve is quite low, as the DM - in its core concept - does not distinguish between function of devices, but only their angular orientation with respect to transmitter. However BER for Bob can be seen equally low, despite Bob missing data required to calculate (1). This allows good communication, as for all configuration BER is consistently better than $10^{-4}$.

Fig $5 \mathrm{~b}$ demonstrates the BER patterns for Eve, when $\phi_{B o b}$ is 


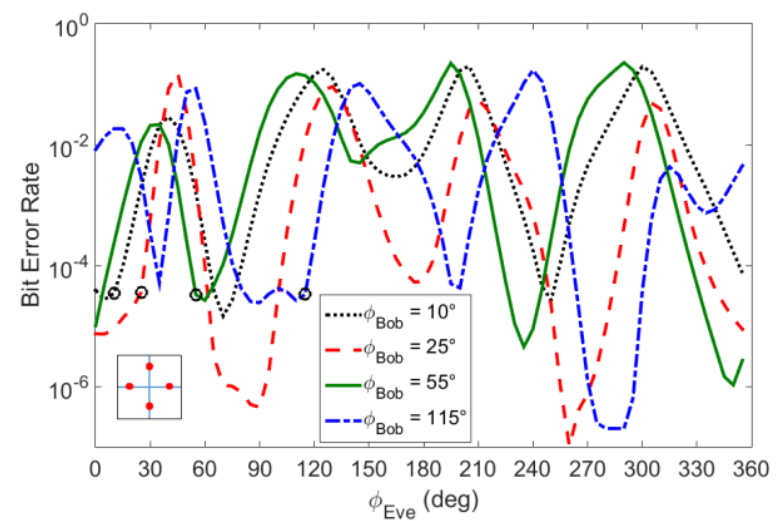

Fig. 6. Eve's BER for the $45^{\circ}$ constellation rotated 4-PSK modulation: as a function of direction $\phi_{\mathrm{Eve}}$ in four exemplary $\phi_{\mathrm{Bob}}$ cases.

constant (shown for four exemplary values) and $\phi_{E v e}$ varies. Although only 4 exemplary configurations of $\phi_{B o b}$ are shown, they are representative for all 24 configurations generated (results not shown for clarity). Due to the assumption that Eve has full system information, the BER values correspond to the worst possible scenario. On each curve, the $\phi_{B o b}$ is marked by a black circle. It can be seen, that once the $\phi_{E v e}$ departs from $\phi_{B o b}$ the BER for Eve increases rapidly. An undesired minimum in BER-pattern can be seen when $\phi_{E v e}$ is approximately a multiplication of $90^{\circ}$ from $\phi_{B o b}$. This is due to the symmetry in this particular antenna design [7] and consequently in the radiation pattern (see Fig. 3).Due to manufacturing inaccuracies however some minima might be deeper than others. Please note however, that a similar problem will occur with classical DM using an antenna array with omnidirectional antennas. For the latter case, the undesired minima will be mirrored with respect to the array plane.

Fig. 6 demonstrates the proposed scheme when the 4-PSK modulation is rotated by $45^{\circ}$ in the modulation plane, i.e. original modulation vectors are located on the I/Q axes. Although having the same information content as standard 4PSK, the scheme might offer some benefits for the design of RF-frontends, as each symbol is transmitted solely by a single radiator. For all cases Bob's BER is kept below $10^{-4}$ and the overall performance is comparable with the results shown in Fig. 5. The proposed scheme offers comparable performance as other reported works, which however use large antenna arrays: [2] reports BER below $10^{-4}$ with $12 \mathrm{~dB}$ SNR and 4 element array

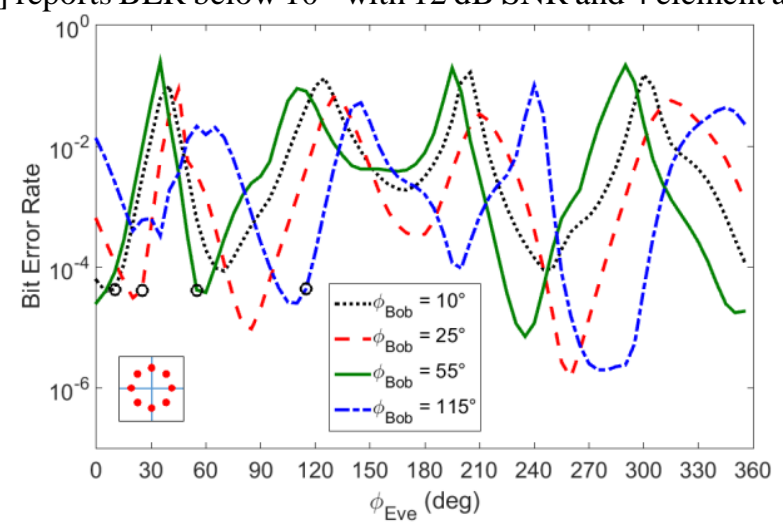

Fig. 7. Eve's BER for the 8-PSK modulation as a function of direction $\phi_{\text {Eve }}$ in four exemplary $\phi_{\mathrm{Bob}}$ cases. of total size $2 \lambda$; [3] reports BER below $10^{-5}$ with $17 \mathrm{~dB}$ SNR and 7 element array of total size $3.5 \lambda$; [6] reports BER below $10^{-3}$ with $8 \mathrm{~dB}$ SNR and the antenna's size is at least $1.5 \lambda$.

\section{B. 8-PSK Modulation}

Fig. 7 shows results for 8-PSK modulation. For this scenario, the SNR level had to be increased to $17 \mathrm{~dB}$ in order to achieve a comparable BER around $10^{-4}$. With minor variations the same BER pattern as the previous cases can be seen, i.e. similar to 4PSK modulation with $12 \mathrm{~dB}$ SNR. This demonstrates, that for channels with better SNR values, one can still maintain comparable privacy levels by using a more information-dense modulation scheme. This additionally increases the communication speed and can be especially useful for future implementations of adaptive OFDM schemes.

\section{CONCLUSION}

The paper proposes a directional modulation scheme, which for the first time uses a compact antenna rather than an extensive array. This is achieved by calculating the weighting scheme using a-priori known complex radiation patterns and an antenna with a sufficiently variable pattern, i.e. with a phase variation across the antenna beam. This enables DM schemes from spatially-constrained platforms, e.g. wireless sensor nodes or future antennas integrated into Internet of Things. It is demonstrated, that information-dense modulation schemes can be used without compromising privacy.

The proposed system is compact and simple, and is expected to only marginally increase the transceiver cost. The solution is considered as a promising candidate to increase privacy for radios operating from small platforms, as required in many "Internet of Things" applications.

\section{REFERENCES}

[1] C. Kolias, A. Stavrou, J. Voas, I. Bojanova, and R. Kuhn, "Learning Internet-of-Things Security "Hands-On"', IEEE Security \& Privacy, vol. 14, no 1, pp. 37-46, Jan-Feb. 2016.

[2] M. P. Daly, E. L. Daly, and J. T Bernhard, "Demonstration of Directional Modulation Using a Phased Array", IEEE Trans. on Ant. and Prop., vol. 58, no 5, pp. 1545-1550, May 2010.

[3] Yuan Ding, and V. Fusco, "Directional modulation-enhanced retrodirective array", Electronics Letters, vol. 51, no 1, pp. 118-120, Jan. 2015.

[4] Yuan Ding, and V. Fusco, "Developments in Directional Modulation Technology", Forum for Electromagnetic Research Methods and Application Technologies (FERMAT), vol. 13, Jan.-Feb. 2016.

[5] A. Narbudowicz, D. Heberling, and M. J. Ammann, "Low-cost directional modulation for small wireless sensor nodes", in: EuCAP - European Conf. on Antennas and Propagation, Davos, Switzerland, Apr. 2016.

[6] Tao Hong, Mao-Zhong Song, and Yu Liu, "Dual-Beam Directional Modulation Technique for Physical-Layer Secure Communication", IEEE Ant. and Wireless Prop. Letters, vol. 10, pp. 1417-1420, Dec. 2011.

[7] A. Narbudowicz, M. J. Ammann, and D. Heberling "Switchless Reconfigurable Antenna with $360^{\circ}$ Steering," IEEE Antennas and Wireless Propagation Letters, vol. 15, pp. 1689-1692, 2016.

[8] A. Narbudowicz, M. J. Ammann, and D. Heberling, "Systematic Approach to Inhomogeneous Linear Arrays", in: 2016 IEEE Symp. on Ant. and Prop. and USNC-URSI Radio Science Meeting, Fajardo, USA, June 2016 